\title{
Characterization of bacterial acylpeptidyl-oligopeptidase
}

Takayuki K. Nemoto*, Toshio Ono, Takeshi Kobayakawa, and Yuko Ohara-Nemoto

*Corresponding author:

Takayuki K. Nemoto, Department of Oral Molecular Biology, Nagasaki University Graduate School of Biomedical Sciences, 1-7-1 Sakamoto, Nagasaki 852-8588, Japan, e-mail: tnemoto@nagasaki-u.ac.jp

Toshio Ono, Takeshi Kobayakawa, and Yuko Ohara-Nemoto: Department of Oral Molecular Biology, Nagasaki University Graduate School of Biomedical Sciences, 1-71 Sakamoto, Nagasaki 852-8588, Japan

Running title: General properties of acylpeptidyl-oligopeptidase

\section{Highlights}

- Acylpeptidyl-oligopeptidase (AOP) liberates N-terminally acylated dipeptides.

- AOP belongs to S09.A77 peptidase distributed in prokaryotes and eukaryotes.

- Bacterial AOP prefers hydrophobic P1 and P2 residues.

- AOP liberates acetyl-Ser ${ }^{1}-\mathrm{Ty}^{2}$ from $\alpha$-melanocyte-stimulating hormone. 


\section{ABSTRACT}

Acylpeptidyl-oligopeptidase (AOP), which has been recently identified as a novel enzyme in a periodontopathic bacterium, Porphyromonas gingivalis, removes di- and tri-peptides from N-terminally acylated polypeptides, with a preference for hydrophobic P1-position amino acid residues. To validate enzymatic properties of AOP, characteristics of two bacterial orthologues from Bacteroides dorei (BdAOP), a Gramnegative intestinal rod, and Lysinibacillus sphaericus (LsAOP), a Gram-positive soil rod, were determined. Like P. gingivalis AOP (PgAOP), two orthologues more efficiently hydrolyzed N-terminal acylated peptidyl substrates than non-acylated ones. Optimal pH was shifted from 7.0 - 8.9 for $\mathrm{N}$-acylated to 8.5 - 9.5 for non-acylated substrates, indicating preference for non-charged hydrophobic N-terminal residues. Hydrophobic P1- and P2-position preferences were common in the three AOPs, although PgAOP preferred Leu and the others preferred Phe at the P 1 position. In vitro mutagenesis demonstrated that $\mathrm{Phe}^{647}$ in PgAOP was responsible for the P1 Leu preference. In addition, bacterial AOPs commonly liberated acetyl-Ser ${ }^{1}-\mathrm{Tyr}^{2}$ from $\alpha$ melanocyte-stimulating hormone. Taken together, although these three bacterial AOPs exhibited some variations in biochemical properties, the present study demonstrated the existence of a group of exopeptidases that preferentially liberates mainly dipeptides from N-terminally acylated polypeptides with a preference for hydrophobic P1 and P2position residues.

\section{Key words}

Acylaminoacyl-peptidase, acylpeptidyl-oligopeptidase, Bacteroides dorei, Lysinibacillus sphaericus, Porphyromonas gingivalis. 


\section{Introduction}

Acylpeptidyl-oligopeptidase (AOP), which has been recently identified as a novel enzyme in a periodontopathic bacterium, Porphyromonas gingivalis, removes di- and tri-peptides from N-terminally acylated polypeptides, with a preference for hydrophobic P1-position residues [1]. Porphyromonas gingivalis is an asaccharolytic Gram-negative obligate anaerobic rod that has been implicated as a causative agent of chronic periodontal disease [2]. Since this bacterium solely utilizes amino acids as energy and carbon sources, exopeptidases producing dipeptides from nutritional oligopeptides are crucial for its growth and pathogenicity [3, 4].

P. gingivalis expresses four different dipeptidyl peptidases (DPPs) in periplasmic space, which liberate N-terminal dipeptides from polypeptides, including DPP4 specific for penultimate Pro [5, 6], DPP5 for Ala and hydrophobic residues [7], DPP7 for hydrophobic residues [8, 9], and DPP11 for acidic residues [10]. Although oligopeptides with most combinations of the N-terminal (P2 position) and penultimate (P1) amino acid residues are degraded by these DPPs, no DPP is capable of managing polypeptides with N-terminally acylated substrates [6, 8, 10]. Thus, P. gingivalis AOP (PgAOP) should be highly beneficial in this organism.

PgAOP (PGN_1349) [11] is composed of 759 amino acids and currently listed as an S9 family class C unassigned peptidase in the MEROPS peptidase database [12]. The KEGG orthology database lists at least 142 PgAOP orthologues in bacteria as well as eukaryotes [13]. In addition, we previously demonstrated that the amino acid sequence of PgAOP is partially related to that of the Caenorhabditis elegans DPP family (DPF)-6 with similarity of $27.3 \%$ [1]. Among these orthologues, however, the peptidase activity of PgAOP has been solely reported to date.

Several types of peptidases that prefer N-terminally blocked oligopeptides have been identified. Both C. elegans DPP-5/mammalian acylaminoacyl-peptidase (AAP) [14 16] and archaea AAP [17, 18] remove N-terminal acylated amino acids from a polypeptide. Pyrococcus furiosus N-acetyl deblocking aminopeptidase/PhTET1 aminopeptidase removes the $\mathrm{N}$-terminal acyl group and successive amino acid residues 
[19], and P. furiosus pyroglutamate aminopeptidase specifically releases $\mathrm{N}$-terminal pyroglutamic acid [20]. However, to our knowledge, no exopeptidase that removes $\mathrm{N}$ terminal acylated di- and tri-peptides from a polypeptide is present other than AOP.

In the present study, to validate general enzymatic properties of AOP, we expressed two additional bacterial orthologues, one from Bacteroides dorei, a Gram-negative intestinal obligate anaerobic rod, and the other from Lysinibacillus sphaericus, a Grampositive facultative anaerobic soil rod. The present findings indicate that AOP should be settled into one exopeptidase family that predominantly liberates acyl-dipeptides with hydrophobic P1- and P2-position residues.

\section{Materials and methods}

\subsection{Bacterial strains and materials}

Genomic DNA of B. dorei JMC13471 [21] and L. sphaericus JCM2502T [22] were provided by RIKEN BRC through the National Bio-Resource Project of MEXT, Japan. Restriction and DNA-modifying enzymes were purchased from Takara Bio (Tokyo, Japan) and New England Biolabs (Ipswich, MA). Quick Taq HS DyeMix came from Toyobo (Tokyo, Japan). $\alpha$-Melanocyte-stimulating hormone ( $\alpha$-MSH), leupeptin, and most peptidyl-4-methycoumaryl-7-amide (MCA) were purchased from the Peptide Institute (Osaka, Japan). Acetyl (Ac)-Ala-, ac-Met-, Gly-Phe-, Ser-Tyr-, and Thr-SerMCA were from Bachem (Bubendorf, Switzerland). Commercially unavailable substrates, i.e., Leu-Leu-, Phe-Leu-, Lys-Met-, benzyloxycarbonyl (Z)-Lys-Met-, and ZAVKM-MCA, were synthesized by Thermo Fisher Scientific (Ulm, Germany) and Scrum (Tokyo, Japan). Peptidase inhibitors except for leupeptin were from SigmaAldrich Japan (Tokyo, Japan).

\subsection{Expression and purification of recombinant proteins}


A DNA fragment encoding Lys ${ }^{2}-$ Lys $^{631}$ of AOP [GV66_05060 (KEGG entry code)] from $B$. dorei was amplified with a set of primers (5'AAAACATTAAAACGCATTTTAGCCGCAAC-3' and 5 TTTTTTCAGGTGAGCATCCAGGAATTTCT-3'). A DNA fragment encoding the mature form of AOP [Lsph_0460 (KEGG entry code), Ser ${ }^{23}$ Lys $^{757}$ ] from L. sphaericus was amplified with 5'-TCCATTCAGGAAGTACGAGCTGCAAATGA-3' and 5'TTtTAAATGGCTGTCGAAGAATTTAACCA-3'. The PCR products were inserted into pTrcHis2 TOPO (Invitrogen, Carlsbad, CA), according to the manufacturer's protocol, resulting in production of pTrcHis2-BdAOP and -LsAOP, respectively. Construction of pTrcHis2-PgAOP encoding PGN_1349 (Met ${ }^{16}$-Lys $^{579}$ ) was previously reported [1].

E. coli XL-1 Blue cells carrying expression plasmids were cultured in Luria-Bertani broth supplemented with $75 \mu \mathrm{g} / \mathrm{ml}$ of ampicillin at $37{ }^{\circ} \mathrm{C}$. Recombinant proteins were induced with $0.2 \mathrm{mM}$ isopropyl-thiogalactopyranoside at $30{ }^{\circ} \mathrm{C}$ for $4 \mathrm{~h}$, then purified using Talon affinity chromatography, as previously reported [7, 10]. In vitro mutagenesis was performed using PCR techniques with appropriate primers to introduce single amino acid substitutions of $\mathrm{Ile}^{583}, \mathrm{Ile}^{644}$, Phe ${ }^{647}$, and $\operatorname{Trp}^{666}$ of PgAOP to Trp ${ }^{583}$, $\mathrm{Leu}^{644}, \mathrm{Leu}^{647}$, and $\mathrm{Val}^{666}$, respectively.

\subsection{Peptidase activity}

Peptidase activity was determined using peptidyl-MCA, as previously reported [7, 10], with the buffers slightly modified. Briefly, the reaction was started by addition of recombinant proteins $(10 \mathrm{ng}-1 \mu \mathrm{g})$ in a reaction mixture $(200 \mu 1)$ composed of $50 \mathrm{mM}$ sodium phosphate ( $\mathrm{pH} 8.0$ ), $5 \mathrm{mM}$ EDTA, and $20 \mu \mathrm{M}$ peptidyl MCA. After 30 min at 37 ${ }^{\circ} \mathrm{C}$, fluorescence intensity was measured with excitation at $380 \mathrm{~nm}$ and emission at 460 $\mathrm{nm}$. For some experiments, $\mathrm{pH}$ values varied from 5.5 - 9.5 with $50 \mathrm{mM}$ sodium phosphate $(\mathrm{pH} 5.5-8.5)$ or Tris- $\mathrm{HCl}(\mathrm{pH} 7.0$ - 10.0). For inhibition studies, AOP was preincubated with an inhibitor for $15 \mathrm{~min}$ at $0{ }^{\circ} \mathrm{C}$, Z-Lys-Met-MCA was added, and then 
residual activity was measured. In some experiments, $\mathrm{NaCl}$ concentration was varied from 0 to $1.6 \mathrm{M}$, or the reaction was performed at $0-70{ }^{\circ} \mathrm{C}$. Values are shown as the average \pm S.D. and calculated from at least three independent measurements.

\subsection{MALDI-TOF MS}

$\alpha$-MSH $(20 \mu \mathrm{M})$ was incubated in $100 \mu 1$ of $50 \mathrm{mM} \mathrm{Na-phosphate} \mathrm{buffer,} \mathrm{pH} \mathrm{7.5,}$ containing $5 \mathrm{mM}$ EDTA in the presence of AOP $(0.1 \mu \mathrm{g})$ at $37{ }^{\circ} \mathrm{C}$ for $0-30 \mathrm{~min}$, then MS analysis was performed as previously reported [23]. The reaction was stopped at the appropriate time point by addition of trifluoroacetic acid $(0.1 \%)$, then hydrolyzed products were adsorbed to a Millipore ZipTip-C18 tip, then washed with $0.1 \%$ trifluoroacetic acid and eluted with $50 \%$ acetonitrile containing $5 \mathrm{mg} / \mathrm{ml}$ of $\alpha$-cyano-4hydroxycinnamic acid. The molecular masses of the products were determined by mass spectrometry using a Bulker Ultraflex III (Billerica, MS).

\subsection{Size-exclusion HPLC}

Recombinant peptidases were subjected to size exclusion HPLC using ÄKTA explorer 10S (GE Healthcare, Chicago, U.S.A.) with a Superdex200 increase 10/300 column $(1.5 \times 30 \mathrm{~cm})$ equilibrated with $20 \mathrm{mM}$ Tris- $\mathrm{HCl}(\mathrm{pH} 8.0)$ containing $0.1 \mathrm{M}$ $\mathrm{NaCl}$. They were eluted with the identical buffer at a rate of $0.5 \mathrm{ml} / \mathrm{min}$ at room temperature, then $0.5-\mathrm{ml}$ fractions were collected. An aliquot $(1 \mu 1)$ of the fractions was subjected to a peptidase assay with Z-Lys-Met-MCA. Blue dextran (void volume), ferritin (430 kDa), aldolase (182 kDa), bovine serum albumin (67 kDa), (GE Healthcare), ovalbumin (45 kDa) (Wako Pure Chemicals, Osaka, Japan), and cytochrome C (12 kDa) (Sigma-Aldrich) were used as molecular standards. 


\section{Results}

\subsection{Comparison of amino acid sequences}

In this study, we chose two bacterial orthologues of PgAOP (PGN_1349) from $B$. dorei (GV66_05060) and L. sphaericus (Lsph_0460), which are tentatively annotated as S9 peptidase and DPP, respectively [12]. The genera Bacteroides and Porphyromonas are phylogenetically close Gram-negative anaerobes, while L. sphaericus is a Grampositive bacterium. Amino acid sequence alignments show that PgAOP has a $38.7 \%$ similarity with GV66_05060 and 34.3\% similarity with Lsph_0460 (Table 1 and Supplemental Fig. S1). Both of them showed higher similarities to C. elegans DPF-6 than to DPF-5. In the present study, GV66_05060 and Lsph_0460 were designated as BdAOP and LsAOP, respectively.

The amino acid similarity is more significant in the C-terminal half, in which three essential residues of serine peptidases, i.e., $\mathrm{Ser}^{615}$, $\mathrm{Asp}^{702}$, and $\mathrm{His}^{734}$ in PgAOP numbering, are conserved. In addition, $\mathrm{Pro}^{537}$ and six hydrophobic residues, which are possibly involved in substrate binding [1], are nearly completely conserved. That is, $\operatorname{Pro}^{537}, \mathrm{Tyr}^{619}$, and $\mathrm{Val}^{641}$ are identical, and both $\mathrm{Ile}^{583}$ and $\operatorname{Tr}^{666}$ are substituted by Trp and Val, respectively, in the two orthologues (see Fig. 3). In addition, Ile ${ }^{644}$ is substituted by Leu in BdAOP and Phe ${ }^{647}$ by Leu in LsAOP. Taken together, though three residues are possibly substituted, the hydrophobic property of the substrate-binding pocket seems to be maintained in these putative AOPs.

\subsection{Determination of optimal $\mathrm{pH}$ and inhibition profile}

Recombinant PgAOP, BdAOP, and LsAOP migrated to the 85-, 65-, and 85-kDa positions, respectively, on SDS-PAGE, reflecting their calculated molecular masses (Supplemental Fig. S2). Their hydrolyzing activities were confirmed using Z-Lys-MetMCA, indicating that the GV66_05060 and Lsph_0460 genes truly encode AOP. 
Since the optimal $\mathrm{pH}$ of PgAOP varies on different substrates, $\mathrm{pH}$ dependence of the activities of the two AOPs were initially examined using $\mathrm{N}$-terminal acylated- and nonacylated-MCA substrates, including glutalyl (Glt)-AAF-, AAF-MCA, Z-Lys-Metand Lys-Met-MCA, as well as Z-VKM-MCA. The optimal $\mathrm{pH}$ value of BdAOP for AAF, Glt-AAF-, and Z-VKM-MCA was 8.7, 7.2, and 7.5, respectively, and of LsAOP for AAF-, Met-Leu-, Lys-Met-, Z-Lys-Met-, and Z-VKM-MCA was 8.5, 9.5, 9.4, 8.9, and 7.8, respectively (Supplemental Fig. S2). These results confirmed that the optimal $\mathrm{pH}$ values of AOP generally vary on the substrates based on the state of N-terminal acylation (Table 2). That is, the optimal $\mathrm{pH}$ for $\mathrm{N}$-acylated substrates ranges from 7.2 (Glt-AAF-MCA) to 8.9 (Z-Lys-Met-MCA) and for non-acylated substrates from 8.5 (AAF-MCA) to 9.5 (Met-Leu-MCA). These results suggest that deionization of an $\mathrm{N}$ terminal amino group enhances the hydrolytic activity.

We next examined the effects of peptidase inhibitors on three AOPs (Table 3). In consistent to the S9-family classification, serine protease inhibitors, such as Pefabloc, 3, 4-dichloroisocoumarin, and phenylmethylsulfonyl fluoride, efficiently blocked the activities with an exception of Pefabloc on BdAOP. Cysteine peptidase inhibitors, i.e., leupeptin, E-64, and monoiodoacetic acid, and a metallopeptidase inhibitor EDTA showed no effect on any AOP. These results suggest that AOPs commonly possess the properties of serine peptidase, but the critical difference in the response to Pefabloc may reflect the structural difference between BdAOP and others.

\subsection{Substrate specificity of AOPS}

The substrate specificities of BdAOP and LsAOP were examined using various peptidyl-MCAs, and then compared with that of PgAOP. Although AOP preferentially hydrolyzes N-terminal-blocked substrates, we used both $\mathrm{N}$-terminal-blocked and unblocked substrates in order to maximize the number of substrates. As shown in Fig. 1, BdAOP exhibited the highest activity against Z-Lys-Met-MCA, followed in order by AAF-, Gly-Phe-MCA, Z-AVKM, and Z-VKM-MCA, whereas LsAOP showed the highest 
activity toward Leu-Leu-MCA, followed in order by Gly-Phe-, AAF-, Met-Leu-, Z-LysMet, and Phe-Leu-MCA. Hydrolysis toward Z-VKM was demonstrated with PgAOP and BdAOP, while it was subtle with LsAOP. Furthermore, the activities of BdAOP and LsAOP toward AAF- and Gly-Phe-MCA were significant, whereas those were low with PgAOP. Hydrolysis toward succinyl (Suc)-LLVY-, Z-HEK-, and Thr-Ser-MCA of the three AOPs was subtle, and each scarcely hydrolyzed Z-Met-MCA, indicating that AOP less potently liberates tri- and tetra-peptides and does not possess aminopeptidase activity.

Despite of the presence of apparent variations in the hydrolyses among three AOPs (Fig. 1A), close positive relationships were observed in their substrate preferences, when the activities of one AOP were plotted against those of the other (Fig. 1B). These results suggest the existence of common properties in the three AOPs beyond apparent variations of the activity.

We previously identified three properties related to the substrate specificity of PgAOP, as follows. (i) PgAOP is preferential for substrates with P1-position hydrophobic residues. (ii) It more potently hydrolyzes di- and tri-peptidyl MCA than aminoacyl and tetrapeptidyl MCA. (iii) It is extremely preferential for acylated substrates as compared to substrates with a free N-terminus. We then systematically examined these factors in detail with BdAOP and LsAOP, as well as PgAOP.

\subsection{P1- and P2-position specificity}

The P1-position preference was investigated using dipeptidyl MCA substrates with Lys at the N-terminal P2 position (Fig. 2A). PgAOP most preferentially hydrolyzed Lys-Leu-MCA, followed by Lys-Ala-MCA, whereas BdAOP solely hydrolyzed Lys-PheMCA. LsAOP predominantly hydrolyzed Lys-Phe-MCA as did BdAOP. Taken together, we concluded that PgAOP prefers Leu at the P1 position, and that BdAOP and LsAOP prefer Phe. Hence, the dominant hydrolysis of AAF- and Gly-Phe-MCA by BdAOP and LsAOP, but not by PgAOP (Fig. 1) can be explained, at least in part, by the different 
P1-position preferences among the three AOPs.

Presently, there are no data available regarding the P2-position specificity of PgAOP. Hence, we examined the role of $\mathrm{P} 2$ amino acid residue by using two dipeptidyl MCAs with P1-position Phe and four substrates with P1 Leu (Fig. 2B). As a result, distinct tendencies of the P2 preference were observed. Hydrolyses of Phe-Leu-, Leu-Leu-, and Met-Leu-MCA by PgAOP were substantially higher than those of Lys-Leu-, Gly-Phe-, and Lys-Phe-MCA. These results suggest that PgAOP prefers hydrophobic P2 residues, while scarcely accept charged and small residues. In contrast, BdAOP obviously hydrolyzed Lys-Phe- and Gly-Phe-MCA reflecting their P1-position Phe preference. The profile of LsAOP was similar to that of BdAOP, except that hydrolysis of Phe/Leu/Met-Leu-MCA was increased to a level similar to that of Gly-Phe-MCA. In consideration of the effect of the P2 residues of the three AOPs, it should be noted that the hydrolysis by three AOPs for Leu-Leu-, Met-Leu-, and Phe-Leu-MCA was more efficient as compared to that of Lys-Leu-MCA indicated that AOPs commonly prefer hydrophobic residues at both the $\mathrm{P} 2$ and $\mathrm{P} 1$ positions.

\subsection{Peptide length of MCA substrates}

Almost no activity was observed with Z-Met-MCA with any AOP, indicating that AOP does not possess an AAP activity. Among Z-Lys-Met-, Z-VKM-, and Z-AVKMMCA, maximal hydrolysis for acyl-dipeptidyl (Z-Lys-Met)-MCA was clearly shown by the three AOPs (Fig. 2C), confirming that AOP more preferentially liberates $\mathrm{N}$-acylated dipeptide from substrates than $\mathrm{N}$-acylated tri- and tetra-peptides. Although we previously reported that PgAOP cleaves di- as well as tri-peptidyl-MCA, the present study confirmed that the most potent liberation of the $\mathrm{N}$-terminal acylated-dipeptide is a general feature of AOP.

\subsection{Effect of N-terminal modification of substrates}


Figure 1 shows hydrolysis of not only N-terminal acylated but also unblocked substrates, such as AAF- and Gly-Phe-MCA, by BdAOP and LsAOP, and that LsAOP further hydrolyzed Leu-Leu-, Phe-Leu-, and Met-Leu-MCA. Accordingly, we examined the effect of N-terminal acylation using four pairs of substrates. As shown in Table 4, the three AOPs commonly showed higher activities for acylated forms of Ala-, Met-, and Lys-Met-MCA, with the magnitude most significant with PgAOP for Ala- and LysMet-MCA. Exceptionally, acylation of AAF-MCA reduced its hydrolysis by BdAOP and LsAOP, in contrast to a 13.6-fold enhancement by PgAOP. These results may be related to findings showing that the best substrate for AOP is acylated-dipeptidyl MCA, with which N-terminal acylation produced maximal enhancement of the activity. That is, the distance of the N-terminal acyl groups in dipeptidyl-MCA substrates may be suitable, while that of tripeptidyl MCA is rather distant from the active center, thus the effect of acylation may be less significant as compared to dipeptidyl-MCA.

\subsection{Amino acid residues responsible for different substrate preferences}

The active triad $\mathrm{Ser}^{615} / \mathrm{Asp}^{702} / \mathrm{His}^{734}$ as well as $\operatorname{Pro}^{537}$ [hydrophobicity index (H.I.) $=$ - 46] [24] and 6 hydrophobic amino acid residues (H.I. = 63 - 100) of PgAOP seem to form the substrate-binding pocket. As shown in Supplemental Fig. S1 and Fig. 3, Ile 583 $\left(\right.$ H.I. = 99) is substituted with $\operatorname{Trp}\left(\right.$ H.I. = 97), while $\operatorname{Trp}^{666}($ H.I. = 97) is substituted with Val (H.I. = 76) in both BdAOP and LsAOP. In addition, Ile ${ }^{644}$ is substituted by Leu $\left(\right.$ H.I. = 97) in BdAOP, and Phe ${ }^{647}$ (H.I. = 100) by Leu (H.I. = 97) in LsAOP. Thus, it seems reasonable to assume that these substitutions may be responsible for the subtle change from Leu to Phe preference seen in BdAOP and LsAOP.

To investigate this possibility, we introduced a single amino acid substitution of Ile583Trp, Ile644Leu, Phe647Leu, or Trp666Val into PgAOP, which then migrated on SDS-PAGE to a position identical to that of the wild entity (data not shown). Among the four amino acid substitutions, the substrate-preference profile of PgAOP Phe647Leu (Fig. 3) resembled that of LsAOP (compare Fig. 2 and Fig. 3). Therefore, the alteration 
of substrate preference between PgAOP and LsAOP can be explained, at least in part, by a single amino acid substitution of Phe647Leu. On the other hand, the profile did not change in PgAOP Ile644Leu and the remaining two substitutions, Ile583Trp and Trp666Val, simply induced a decrease in activity of PgAOP (data not shown).

\subsection{Degradation of bioactive peptide $\alpha-M S H$}

We analyzed the degradation of an N-terminally acylated bioactive peptide, $\alpha$-MSH (Ac-S ${ }^{1}$ YSMEHFRWGKPV ${ }^{13}-\mathrm{NH}_{2}, M_{\mathrm{r}}=1664.9$ ), by the three bacterial AOPs using MALDI-TOF MS analysis. As shown in Fig. 4, each of the AOPs produced a single peak at the position of $\mathrm{S}^{3} \mathrm{YSMEHFRWGKPV}{ }^{13}-\mathrm{NH}_{2}\left(M_{\mathrm{r}}=1371.5\right)$, indicating hydrolysis at the $\mathrm{Tyr}^{2}-\mathrm{Ser}^{3}$ bond. Hydrolysis was fast in BdAOP and LsAOP, while it was substantially slow in PgAOP. This difference may be due to structural similarity of Tyr to Phe, which is more preferable to LsAOP and BdAOP at the P1 position, while PgAOP prefers Leu (Fig. 3). In addition, it should be noted that further degradation at the Met ${ }^{4}-\mathrm{Glu}^{5}$ bond did not occur in any of the AOPs, although Met could be located at the P1 position (Fig. 1). This finding confirmed that $\mathrm{N}$-terminally nonacylated dipeptidyl-MCA is scarcely cleaved by AOP.

\subsection{Optimal temperature and molecular state}

When enzymatic reactions were performed at a wide range of temperature for 30 min, the optimal temperatures of PgAOP, BdAOP, and LsAOP were determined to 50, 45 , and $35{ }^{\circ} \mathrm{C}$, respectively (Supplemental Fig. S3). BdAOP showed relatively higher activities at a wide range of temperature $\left(0-45^{\circ} \mathrm{C}\right)$. Three AOPs showed maximal activities without $\mathrm{NaCl}$, and then the activities were gradually decreased at higher salt concentrations (data not shown).

We previously reported that endogenous PgAOP is present as a monomer, while recombinant form predominantly forms oligomers with a minor population of a 
monomer [1]. As shown in Supplemental Fig. S4, size-exclusion HPLC showed that BdAOP was predominantly present as a monomer with a slight amount of dimers (Fractions $26-28$ ). On the other hand, LsAOP was mainly present as a dimer with a minor population of oligomers (Fractions $23-25$ ). These results suggest that AOP has a tendency of oligomerization, of which potential is maximal in PgAOP, followed by LsAOP and BdAOP. Finally, it is important to note that the enzymatic activity of AOP is present independent of their molecular states.

\section{Discussion}

This study presents the biochemical and enzymatic properties of two additional bacterial AOPs from B. dorei and L. sphaericus, as well as those of PgAOP. Although several distinct properties regarding optimal temperature and the molecular states of recombinant forms were observed, the positive relationships of substrate preferences between their activities (Fig. 1) strongly suggested the presence of common characteristics in their peptidase activities. Truly, the common properties of bacterial AOPs are: the P1-position hydrophobic preference, faster liberation of $\mathrm{N}$-terminally acylated dipeptides as compared to acyl-tri- and tetra-peptides, existence of multiple $\mathrm{pH}$ optimums dependent on substrates, and N-terminal acylation-dependent enhancement of peptidase activity. Moreover, it was revealed that these AOPs prefer the hydrophobic P2-position.

The hydrolysis efficiency of the three AOPs was commonly enhanced by N-terminal acylation and their optimal $\mathrm{pH}$ was shifted from neutral for $\mathrm{N}$-acylated substrates to weak alkaline $\mathrm{pH}$ for non-acylated substrates. Our previous 3-D modeling study indicated that PgAOP forms a substrate-binding pocket surrounded by Pro ${ }^{537}$ and 6 hydrophobic residues [1], and the hydrophobicity of the 6 residues is maintained in BdAOP and LsAOP (Fig. 3). In accordance with the hydrophobic characteristics of the active pocket, findings of the present study demonstrated that AOP as well as archaea 
AAP from Aeropyrum pernix K1 [18] prefers hydrophobic residues at the P1 and P2 position. The N-terminal acetyl-, Z-, and Glt-groups attached to the substrates commonly and strongly enhance their activities. Hence, the type of acyl groups is not essential, but blocking of the $\mathrm{N}$-terminal $\alpha$-amino group is critical. Thus, it is reasonable to speculate that a positively charged $\mathrm{N}$-terminus is scarcely acceptable, while an acylated one is suitable for the hydrophobic active pockets of AOP.

Distinct optimal pH levels with acylated and nonacylated substrates can also be explained by the hydrophobic nature of the N-terminus for adaption to the pocket. This is because the $\mathrm{N}$-terminal $\alpha$-amino group is predominantly present in a protonated state at $\mathrm{pH}$ 7.0, then the transition to a non-protonated hydrophobic state at alkaline $\mathrm{pH}$. Thus, an alkaline shift of the optimal $\mathrm{pH}$ that occurs in non-acylated substrates has the same effect as $\mathrm{N}$-terminal acylation. An alkaline shift from optimal $\mathrm{pH}$ for non-acylated substrates was also observed in a study of archaea AAP [18], indicating the same mechanism.

The present study demonstrated that bacterial AOPs hydrolyzed $\alpha$-MSH at the $\mathrm{Tyr}^{2}$ $\mathrm{Ser}^{3}$ bond, and the hydrophobic P1 Tyr (H.I. = 63) is compatible with the preference of AOP. In addition, no further degradation under the present conditions despite the presence of $\mathrm{Met}^{4}($ H.I. $=74)$ further suggested the necessity of N-terminal acylation of substrates for AOP.

It has been reported that archaea AAP shows endopeptidase activity [18], while we also found PgAOP to have such activity [1]. However, since the hydrolyzing efficiency toward tri- and tetra-peptidyl-MCA was significantly lower as compared to dipeptidylMCA, endopeptidase activity of AOP was expected to be absent or at most subtle. We speculated that such endopeptidase activity is only exerted under special conditions, such as when a peptide with an appropriate hairpin structure and a hydrophobic residue is accepted at the substrate-binding pocket of AOP and AAP.

In conclusion, we propose that AOP belongs to S09.A77, in which DPF-6 is the representative member, and possesses common hydrophobic preferences at the P1 and P2 positions, despite that there is a variation in preferences in the P1-position 
hydrophobic residues. The activity is commonly increased by peptidyl substrates harboring an acylated non-charged hydrophobic N-terminus (P2 position). The scarce liberation of the N-terminal acylated amino acid strongly suggests its distinction from

mammalian APP (S09.004) and Aeropyrum-type AAP (S09.A70), although a direct comparison study between AOP and AAP should be indispensable for the conclusion.

\section{Acknowledgments}

This study was supported by a JSPS KAKENHI grant (number JP16K11481). 


\section{References}

[1] T.K. Nemoto, Y. Ohara-Nemoto, G.A. Bezerra, Y. Shimoyama, S.A. Kimura, Porphyromonas gingivalis periplasmic novel exopeptidase, acylpeptidyl oligopeptidase, releases $\mathrm{N}$-acylated di- and tripeptides from oligopeptides. J. Biol. Chem. 291 (2016) 5913-5925.

[2] S.C. Holt, J.L. Ebersole, Porphyromonas gingivalis, Treponema denticola, and Tannerella forsythia: the 'red complex', a prototype polybacterial pathogenic consortium in periodontitis. Periodontology 200038 (2005) 72-122.

[3] N. Takahashi, T. Sato, Preferential utilization of dipeptides by Porphyromonas gingivalis. J. Dent. Res. 80 (2001) 1425-1429.

[4] T.K. Nemoto, Y. Ohara-Nemoto, Exopeptidases and gingipains in Porphyromonas gingivalis as prerequisites for its amino acid metabolism. Jpn. Dental Sci. Rev. 52 (2016) 22-29.

[5] Y. Abiko, Y., M. Hayakawa, S. Murai, H. Takiguchi, Glycylprolyl dipeptidylaminopeptidase from Bacteroides gingivalis. J. Dent. Res. 64 (1985) 106-111.

[6] A. Banbula, A., Bugno, M., Goldstein, J., Yen, J., Nelson, D., Travis, J., et al., Emerging family of proline-specific peptidases of Porphyromonas gingivalis: purification and characterization of serine dipeptidyl peptidase, a structural and functional homologue of mammalian prolyl dipeptidyl peptidase IV. Infect. Immun. 68 (2000) 1176-1182.

[7] Y. Ohara-Nemoto, S.M. Rouf, M. Naito, A. Yanase, F. Tetsuo, T. Ono, et al., Identification and characterization of prokaryotic dipeptidyl-peptidase 5 from Porphyromonas gingivalis. J. Biol. Chem. 289 (2014) 5436-5448. 
[8] A. Banbula, J. Yen, A. Oleksy, P. Mak, M. Bugno, J. Travis, J., et al., Porphyromonas gingivalis DPP-7 represents a novel type of dipeptidylpeptidase. J. Biol. Chem. 276 (2001) 6299-6305.

[9] S.M. Rouf, Y. Ohara-Nemoto, T. Hoshino, T. Fujiwara, T. Ono, T.K. Nemoto, Discrimination based on Gly and Arg/Ser at Position 673 between dipeptidyl-peptidase (DPP) 7 and DPP11, widely distributed DPPs in pathogenic and environmental Gramnegative bacteria. Biochimie 95 (2013) 824-832.

[10] Y. Ohara-Nemoto, Y. Shimoyama, S. Kimura, A. Kon, H. Haraga, T. Ono, et al., Asp- and Glu-specific novel dipeptidyl peptidase 11 of Porphyromonas gingivalis ensures utilization of proteinaceous energy sources. J. Biol. Chem. 286 (2011) 38115 38127.

[11] M. Naito, H. Hirakawa, A. Yamashita, N. Ohara, M. Shoji, H. Yukitake, et al., Determination of the genome sequence of Porphyromonas gingivalis strain ATCC 33277 and genomic comparison with strain W83 revealed extensive genome rearrangements in P. gingivalis. DNA Res. 15 (2008) 215-225.

[12] N.D. Rawlings, M. Waller, A.-J. Barrett, A.-J., A. Bateman, MEROPS: the database of proteolytic enzymes, their substrates and inhibitors. Nucleic Acids Res. 42 (2014) D503-D509.

[13] M. Kanehisa, M. Furumichi, M. Tanabe, Y. Sato, K. Morishima, KEGG: new perspectives on genomes, pathways, diseases and drugs. Nucleic Acids Res. 45 (2017) D353-D361.

[14] S. Tsunesawa, K. Narita, K. Ogata, Purification and properties of acylamino acidreleasing enzyme from rat liver. J. Biochem. 77 (1975) 89-102.

[15] W. Gade, J.L. Brown, Purification and partial characterization of $\alpha$-N-acylpeptide hydrolase from bovine liver. J. Biol. Chem. 253 (1978) 5012-5018.

[16] W.M. Jones, J.M. Manning, Acylpeptide hydrolase activity from erythrocytes. Biochem. Biophys. Res. Commun. 126 (1985) 933-940. 
[17] M. Bartlman, G. Wang, H. Yang, R. Gao, X. Zhao, G. Xie, et al., Crystal structure of an acylpeptide hydrolase/esterase from Aeropyrum pernix K1. Structure 12 (2004) $1481-1488$.

[18] A.L. Kiss, B. Hornung, K. Rádi, Z. Gengeliczki, B. Sztáray, T. Juhász, et al., The acylaminoacyl peptidase from Aeropyrum pernix K1 thought to be an exopeptidase displays endopeptidase activity. J. Mol. Biol. 368 (2007) 509-520.

[19] S. Tsunasawa, Purification and application of a novel N-terminal deblocking aminopeptidase (DAP) from Pyrococcus furiosus. J. Protein Chem. 17 (1998) 521-522.

[20] J. Mozdzanowski, J. Bongers, J., Anumula, K. High-yield deblocking of amino termini of recombinant immunoblobulins with pyroglutamate aminopeptidase. Anal. Biochem. 260 (1998) 183-187.

[21] M.A. Bakir, M. Sakamoto, M. Kitahara, M. Matsumoto, Y. Benno, Bacteroides dorei sp. nov., isolated from human faeces. Int. J. Syst. Evol. Microbiol. 56 (2006) 1639-1643.

[22] I. Ahmed, A. Yokota, A. Yamazoe, T. Fujiwara, Proposal of Lysinibacillus boronitolerans gen. nov. sp. nov., and transfer of Bacillus fusiformis to Lysinibacillus fusiformis comb. nov. and Bacillus sphaericus to Lysinibacillus sphaericus comb. nov. Int. J. Syst. Evol. Microbiol. 57 (2007) 1117-1125.

[23] Y. Ohara-Nemoto, M. Nakasato, Y. Shimoyama, T.T. Baba, T. Kobayakawa, T. Ono, et al., Degradation of incretins and modulation of blood glucose levels by periodontopathic bacterial dipeptidyl peptidase 4. Infect Immun. 85 (2017) pii: e0027717.

[24] O.D. Monera, T.J. Sereda, N.E. Zhou, C.M. Kay, R.S. Hodges, Relationship of side chain hydrophobicity and $\alpha$-helical propensity on the stability of the single-stranded amphipathic $\alpha$-helix. J. Pept. Sci. 1 (1995) 319-329. 


\section{Table 1}

Amino acid sequence comparison of three AOPs and C. elegans DPF-5 and DPF-6.

\begin{tabular}{lllll}
\hline Peptidase & $\begin{array}{l}\text { GV66_05060 } \\
\text { (BdAOP) }\end{array}$ & $\begin{array}{l}\text { Lsph_0460 } \\
\text { (LsAOP) }\end{array}$ & DPF-5 $^{\text {a }}$ & DPF-6 $^{\text {b }}$ \\
\hline PGN_1349 (PgAOP) & 38.67 & 34.26 & 15.06 & 20.27 \\
GV66_05060 & & 47.39 & 14.10 & 23.45 \\
(BdAOP) & & & \\
Lsph_0460 (LsAOP) & & 15.06 & 19.59 \\
DPF-5 & & & 12.21 \\
\hline
\end{tabular}

a and b, C. elegans DPF-5 (MER0004920) and DPF-6 (MER0001920), respectively. 


\section{Table 2}

Optimal pH of three AOPs with various MCA substrates.

\begin{tabular}{lcccccc}
\hline $\begin{array}{l}\text { Peptidas } \\
\text { e Z-VKM- }\end{array}$ & Glt-AAF- & Z-Lys-Met- & AAF- & \multicolumn{2}{l}{ Met-Leu- } & Lys-Met- \\
\hline PgAOP & $7.0^{\mathrm{a}}$ & n.d. & n.d. & n.d. & $8.5^{\mathrm{a}}$ & n.d. \\
BdAOP & 7.4 & 7.2 & n.d. & 8.7 & n.d. & n.d. \\
LsAOP & 7.8 & n.d. & 8.9 & 8.5 & 9.5 & 9.4 \\
\hline
\end{tabular}

Optimal pH determined in Supplemental Fig. S2 is summarized. ${ }^{a}$ Nemoto et al., 2016 [1]. n.d., not determined 
Table 3

Effect of reagents on AOP activities.

\begin{tabular}{|c|c|c|c|c|}
\hline \multirow[b]{2}{*}{ Inhibitor } & \multirow[b]{2}{*}{ Concentration } & \multicolumn{3}{|c|}{ Residual activity (\%) } \\
\hline & & PgAOP & BdAOP & LsAOP \\
\hline $\begin{array}{l}\text { Phenylmethylsulfonyl } \\
\text { fluoride }\end{array}$ & $1 \mathrm{mM}$ & 1 & 0 & 5 \\
\hline $3,4-$ & $1 \mathrm{mM}$ & & & \\
\hline Dichloroisocoumarin & & 6 & 5 & 6 \\
\hline Pefabloc & $8 \mathrm{mM}$ & 19 & 183 & 11 \\
\hline E-64 & $3 \mu \mathrm{M}$ & 101 & 116 & 101 \\
\hline Iodoacetic acid & $5 \mathrm{mM}$ & 73 & 82 & 73 \\
\hline Leupeptin & $0.1 \mathrm{mg} / \mathrm{ml}$ & 99 & 106 & 99 \\
\hline Aprotinin & $0.5 \mathrm{KU}$ & 99 & 120 & 99 \\
\hline EDTA & $10 \mathrm{mM}$ & 97 & 119 & 97 \\
\hline
\end{tabular}




\section{Table 4}

Effects of N-terminal modification of substrates on AOP activities.

\begin{tabular}{|c|c|c|c|c|c|c|}
\hline \multirow[b]{2}{*}{ MCA peptide } & \multicolumn{2}{|c|}{$\mathrm{PgAOP}$} & \multicolumn{2}{|c|}{$\mathrm{BdAOP}$} & \multicolumn{2}{|c|}{ LsAOP } \\
\hline & $\begin{array}{l}\text { Activity }^{\mathrm{a}} \\
(\mathrm{pmol} / \mathrm{min} / \mu \mathrm{g})\end{array}$ & fold increase ${ }^{b}$ & $\begin{array}{l}\text { Activity } \\
(\mathrm{pmol} / \mathrm{min} / \mu \mathrm{g})\end{array}$ & fold increase $\mathrm{e}^{\mathrm{b}}$ & $\begin{array}{l}\text { Activity } \\
(\mathrm{pmol} / \mathrm{min} / \mu \mathrm{g})\end{array}$ & fold increase $\mathrm{e}^{\mathrm{b}}$ \\
\hline Ala- & $0.04 \pm 0.01$ & & $0.028 \pm 0.01$ & & $0.01 \pm 0.00$ & \\
\hline Ac-Ala- & $0.40 \pm 0.01$ & 9.2 & $0.13 \pm 0.00$ & 4.8 & $0.01 \pm 0.00$ & 1.1 \\
\hline Met- & $0.01 \pm 0.01$ & & $0.0 \pm 0.01$ & & $0.43 \pm 0.03$ & \\
\hline Ac-Met- & $0.24 \pm 0.02$ & 21.5 & $0.17 \pm 0.02$ & n. c. & $3.41 \pm 0.09$ & 64.0 \\
\hline Lys-Met- & $0.05 \pm 0.16$ & & $0.09 \pm 0.01$ & & $2.41 \pm 0.09$ & \\
\hline Z-Lys-Met- & $362.17 \pm 1.13$ & 7705.7 & $18.49 \pm 0.60$ & 197.9 & $116.03 \pm 2.29$ & 46.2 \\
\hline \multirow[t]{2}{*}{ AAF - } & $2.54 \pm 0.13$ & & $11.27 \pm 0.40$ & & $311.91 \pm 6.51$ & \\
\hline & & 13.6 & & 0.8 & & 0.04 \\
\hline Glt-AAF- & $34.58 \pm 0.13$ & & $9.06 \pm 0.25$ & & $12.80 \pm 0.42$ & \\
\hline
\end{tabular}

n.c., not calculated, because no activity for Met-MCA was detected. ${ }^{a}$ Nemoto et al., 2016 [1]. ${ }^{b}$ As compared with activity of respective substrates without $\mathrm{N}$-terminal modification. 
A

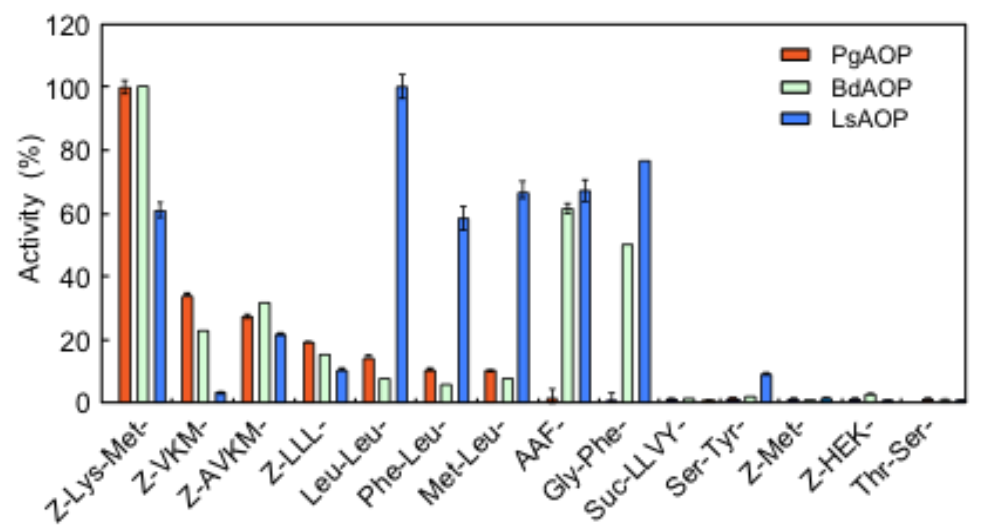

B

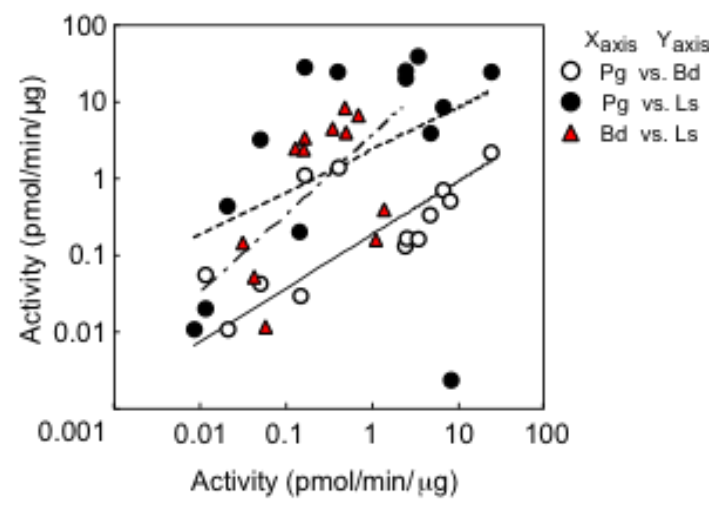

Fig. 1. Substrate specificities of bacterial AOPs.

(A) The activities of PgAOP, BdAOP, and LsAOP were determined with various peptidyl MCAs. The activities of PgAOP (24.6 $\pm 0.8 \mathrm{pmol} / \mathrm{min} / \mu \mathrm{g})$ and $\mathrm{BdAOP}(3.2$ $\pm 0.1 \mathrm{pmol} / \mathrm{min} / \mu \mathrm{g})$ for Z-Lys-Met-MCA, and that of LsAOP $(37.2 \pm 1.4$ $\mathrm{pmol} / \mathrm{min} / \mu \mathrm{g}$ ) for Leu-Leu-MCA were determined to be $100 \%$. Values are shown as the mean \pm S.D. $(n=3)$. (B) Activities of the three AOPs were re-plotted in two combinations along an X-Y axis. Regression lines were calculated for PgAOP vs. BdAOP with $R^{2}=0.52, p=0.004$ (line), PgAOP vs. LsAOP with $R^{2}=0.20, p=0.11$ (dotted line), and BdAOP vs. LsAOP with $R^{2}=0.40$ ( $p=0.04$, broken line). 
A
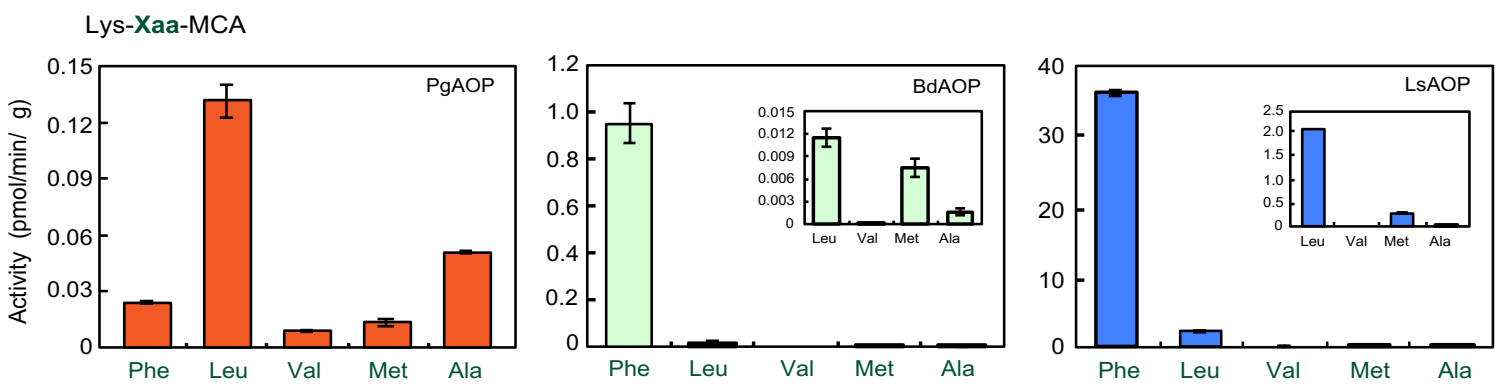

B
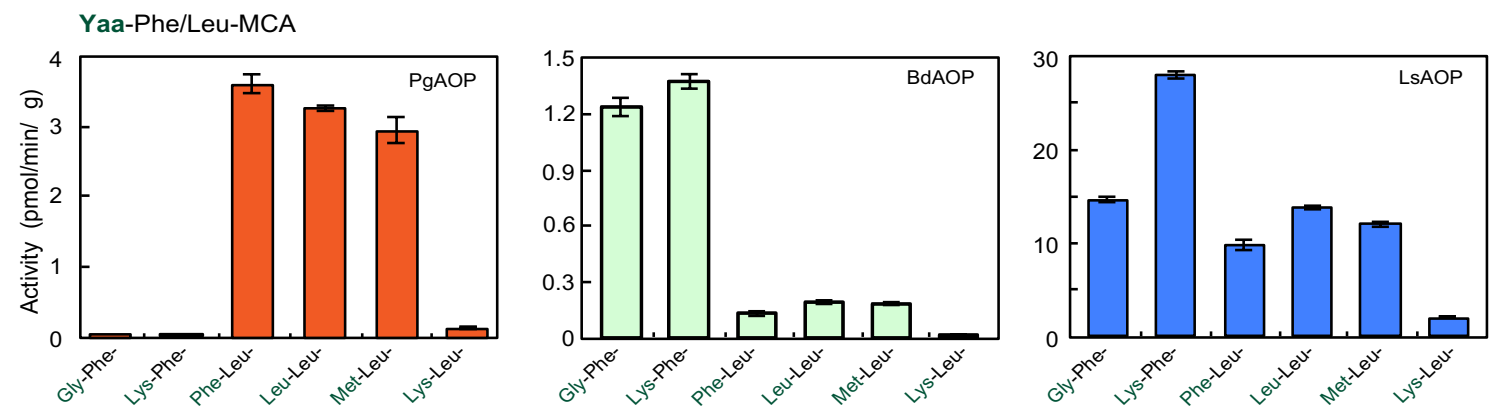

C
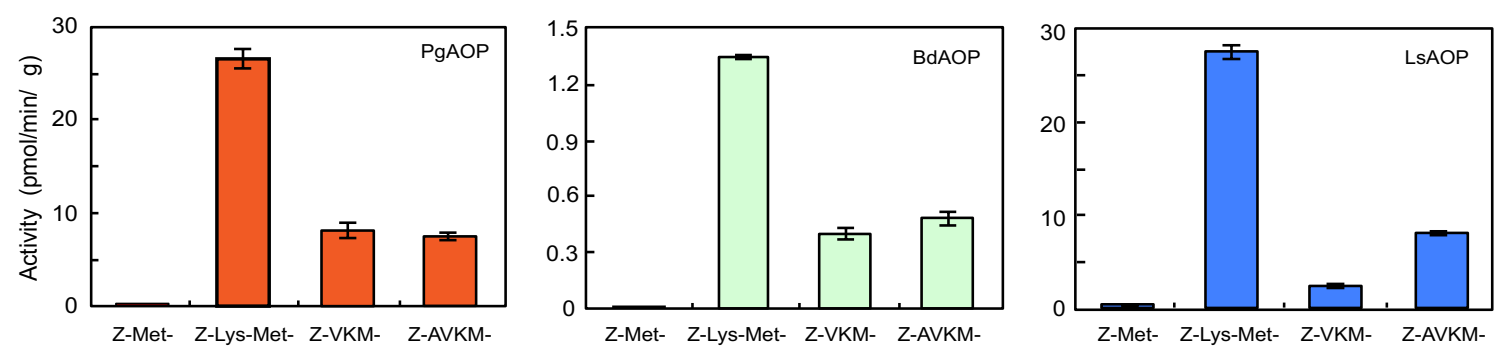

Fig. 2. Effects of P1- and P2-position residues, and substrate length on activity of AOPs. (A) P1-position specificities of PgAOP, BdAOP, and LsAOP were examined with Lys-Xaa-MCA. (B) P2-position specificities were examined with Yaa-Phe- or Yaa-Leu-MCA. (C) The effect of the amino acid length of peptidyl-MCA on the three AOPs was determined. Values are shown as the mean \pm S.D. $(n=3)$ of representative results from three different experiments. 
A

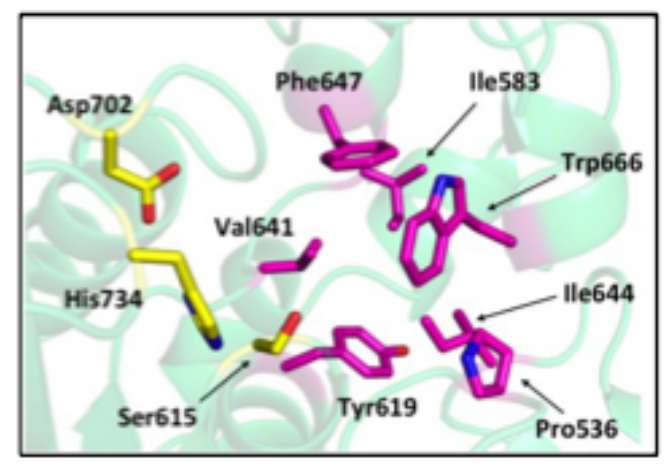

B

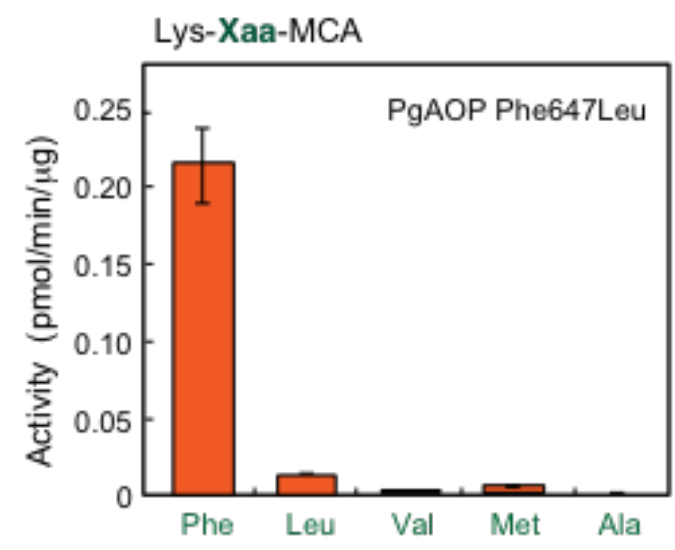

\begin{tabular}{cccc}
\hline a.a. No. & PgAOP & BdAOP & LsAOP \\
\hline 583 & lle (99) & Trp (97) & Trp (97) \\
644 & lle (99) & Leu (97) & Ile (99) \\
647 & Phe (100) & Phe (100) & Leu (97) \\
666 & Trp (97) & Val (76) & Val (76) \\
\hline
\end{tabular}

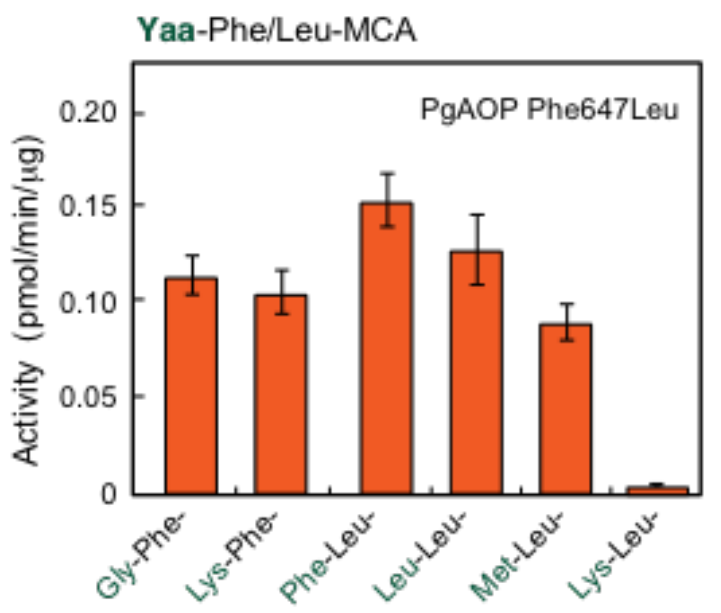

Fig. 3. Effects of amino aid substitutions on substrate specificity of PgAOP.

(A) Stick representation of catalytic triad of serine peptidase (yellow) and the residues forming the $\mathrm{S} 1$ subsite (magenta) in PgAOP [1]. Four amino acid residues potentially responsible for alterations of substrate specificity of BdAOP and LsAOP are presented with H.I. (B) Peptidase activities of PgAOP Phe647Leu were determined with Lys-Xaa-MCA and Yaa-Phe/Leu-MCA. Values are shown as the mean \pm S.D. $(n=3)$ of representative results from three different experiments. 
A
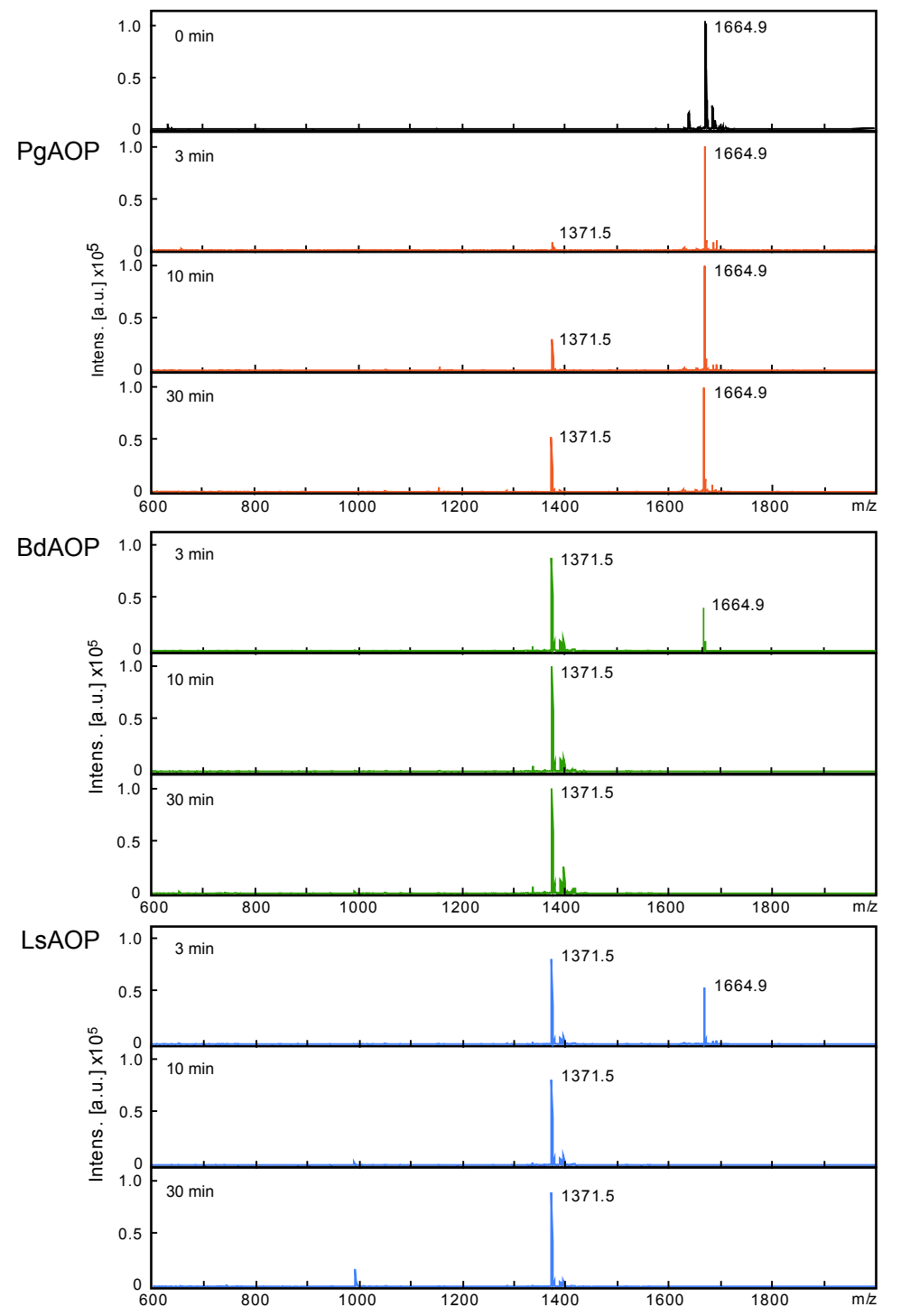

B

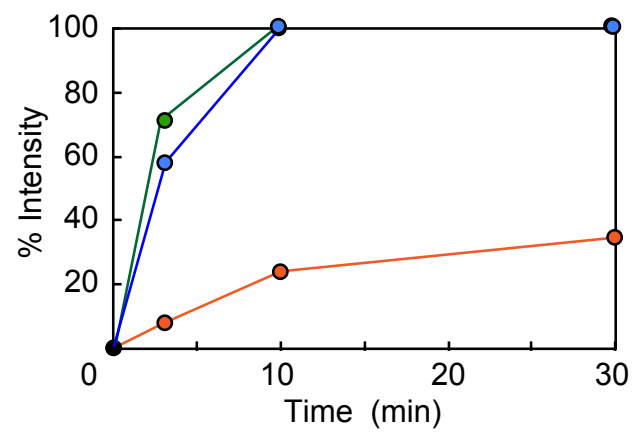

Fig. 4. Degradation of $\alpha-M S H$ by bacterial AOPs. (A) MALDI-TOF MS profiles of $\alpha$-MSH $(0.1 \mu \mathrm{g})$ incubated with $0.1 \mu \mathrm{g}$ of PgAOP, BdAOP, or LsAOP for 0, 3, 10 , and 30 min. (B) Plots showing percent amounts of the product $\left(\mathrm{Ser}^{3}-\mathrm{Val}^{13}-\mathrm{NH}_{2}\right)$ of $\alpha$-MSH following incubation with PgAOP (orange), BdAOP (green), or LsAOP (blue). 


\section{Supplemental Data}

\section{Characterization of bacterial acylpeptidyl-oligopeptidase}

Takayuki K. Nemoto*, Toshio Ono, Takeshi Kobayakawa, and Yuko Ohara-Nemoto

\section{Supplemental References}

[25] A. Scaloni, W.M. Jones, D. Barra, M. Pospischil, S. Sassa, A. Popowicz, et al., Acylpeptide hydrolase: inhibitors and some active site residues of the human enzyme. J. Biol. Chem. 267 (1992) 3811-3818.

[26] H. Wright, A.L. Kiss, Z. Szeltner, L. Polgár, V. Fülöp, Crystallization and preliminary crystallographic analysis of porcine acylaminoacyl peptidase. Acta Crystallogr. Sect. F Struct. Biol. Cryst. Commun. 61 (2005) 942-944. 


\section{Supplemental Table S1}

Comparison of biochemical properties of AOP, mammalian AAP, and archaea AAP (Aeropyrum-type).

\begin{tabular}{|c|c|c|c|}
\hline Subject & $\mathrm{AOP}$ & \multicolumn{2}{|c|}{ AAP } \\
\hline $\operatorname{Name}(\mathrm{s})$ & Acylpeptidyl oligopeptidase & \multicolumn{2}{|c|}{$\begin{array}{l}\text { Acylpeptide hydrolase } \\
\text { Acylaminoacyl peptide hydrolase } \\
\text { Acylamino acid-releasing enzyme }\end{array}$} \\
\hline Species & $\begin{array}{l}\text { Bacteria } \\
\text { P. gingivalis }[1] \\
\text { B. dorei } \\
\text { L. sphaericus }\end{array}$ & $\begin{array}{l}\text { Mammal } \\
\text { human }[16,25] \\
\text { porcine }[26]\end{array}$ & $\begin{array}{l}\text { Archaea } \\
\text { Aeropyrum pernix }[17,18]\end{array}$ \\
\hline $\begin{array}{l}\text { Number of } \\
\text { amino acids }\end{array}$ & $631-759$ & 732 & 582 \\
\hline MEROPS Class & S09.A 77 & S09.004 & S09.A70 \\
\hline Specificity & & & \\
\hline P1-position & hydrophobic & small side chains & large non-polar \\
\hline P2-position & hydrophobic & $\mathrm{n} \cdot \mathrm{d}$ & hydrophobic \\
\hline $\begin{array}{l}\text { Substrate } \\
\text { acylaminoacyl }\end{array}$ & no hydrolysis & Ac-Ala- $p$-nitroanilide ${ }^{c}$ & $\begin{array}{l}\text { Ac-Phe-2-naphthylamide } \\
\text { Ac-Phe- } p \text {-nitroanilide }\end{array}$ \\
\hline acylpeptidyl & acyl-dipeptidyl-MCA & no hydrolysisc & $\begin{array}{l}\text { 2-aminobenzoyl }(\mathrm{Abz})- \\
\mathrm{GF} / \mathrm{EPE}\left(\mathrm{NO}_{2}\right) \mathrm{RA} \\
\mathrm{Abz}-\mathrm{GF} / \mathrm{RPF}\left(\mathrm{NO}_{2}\right) \mathrm{RA} \\
\mathrm{Abz}-\mathrm{EALF} / \mathrm{QGPF}\left(\mathrm{NO}_{2}\right) \mathrm{A}\end{array}$ \\
\hline native peptide & $\alpha-\mathrm{MSH}$ & $\alpha-\mathrm{MSH}$ & $\alpha-\mathrm{MSH}$ \\
\hline
\end{tabular}

n.d., not determined. ${ }^{a}$ present study. 
--MKTLKRILAATTLLVTLGFASEANAQVPLENFFKNPEKAGYQISPDGKYFSYMAPYEN-RLNLFVQEVGSDKATRITSETVRDLAGSMWANGHRILYIKDTAGDEN

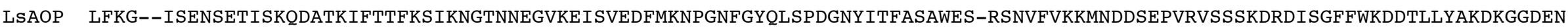

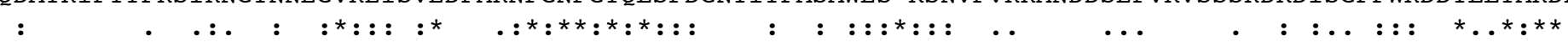

$$
250
$$

300

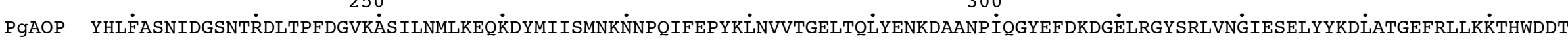
FQLYGVNVDGTDSKAYTAFPKVRTTIIDPLENIDSLVIIGLNKRNPQVFDPYRLNLNNGELTQLAENP---GNIQGWMTDHNGKLRVALAIVDGVNTQILYRETEEQPFRPVLTTNFKET FHIYSSSFNGSEEKDLTPYPGVTVGVLSNLQGVKDEILIMMNKEDAKVFDVYKLNVKTGETKHVAKNP---GNVSNWVADRDGNIRIAVVSDG-VEGTILYRETEKDEFKPF IEMAAGDE

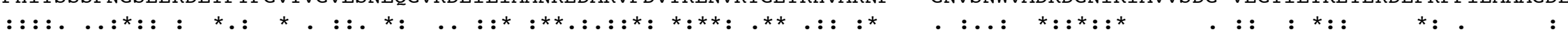
350

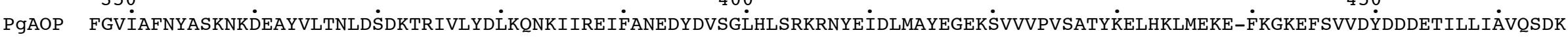

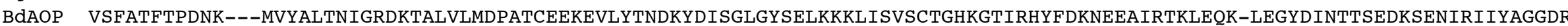
VNPLAFSKDNK---YIYATSNKGRDKVQVVKYDLEGKEE--VIMSNDEVDVAAVLYNSEKDKILYGAYMTDKVHYQFFDEDFEKLFRKIQNKLGVEESELGINDYNKEMTKF IVSVSSDT

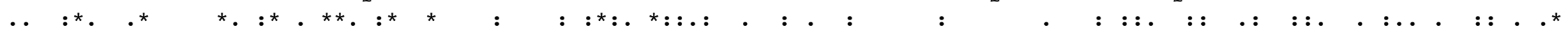
500 550

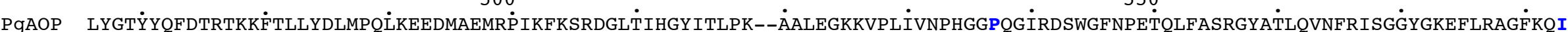

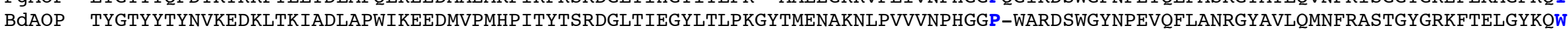

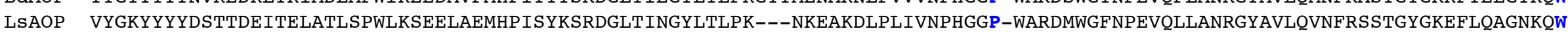

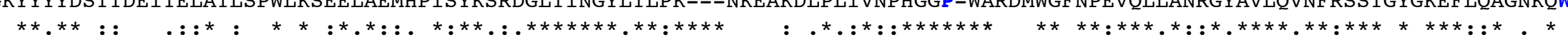
600

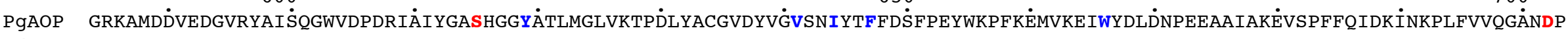

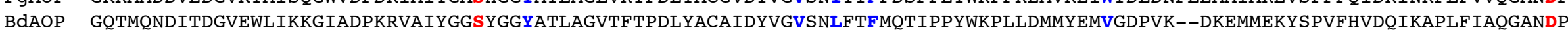

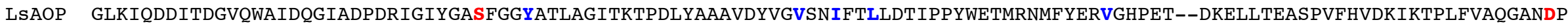

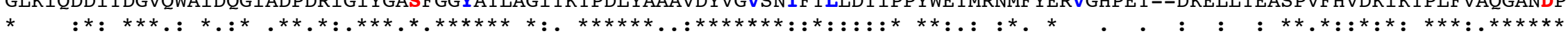

$$
750
$$

PgAOP RVNINESंDQIVTALRAं் GFEVPYMVKY்NEGHGFHREE்NSMELYRAML்GFFAKHLKK BdAOP RVNKAESDQMVEALKKRGIEVEYMVKDNEGHGF HNEENKFDFYRAMEKFLDAHLKK LSAOP RVNQAESDQIVEALRARGVEVEYMLKENEGHGFQNEENRIEFYNAMVKFFDSHLKK $* * * \quad * * * *: * * *: * * . * * * *: * * * * * * *: . * * *::: * . * * \quad *: \quad * * * *$ 
Supplemental Fig. S1. Alignment of amino acid sequences of 3 AOPs.

The amino acid sequences of PgAOP, BdAOP, and LsAOP are aligned. The amino acid numbers of PgAOP are presented. Identical amino acids are indicated by asterisks, and similar amino acids by colons and dots. Ser ${ }^{615}$, Asp ${ }^{702}$, and His ${ }^{734}$ forming the essential triad of serine peptidases are indicated by bold red letters. Potential amino acids forming the substrate-binding pocket of PgAOP [1], and corresponding residues of $\mathrm{BdAOP}$ and LsAOP are indicated by bold blue letters (see Fig. 3A for details). 
A

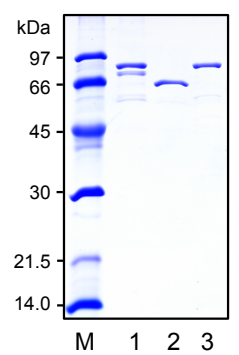

B
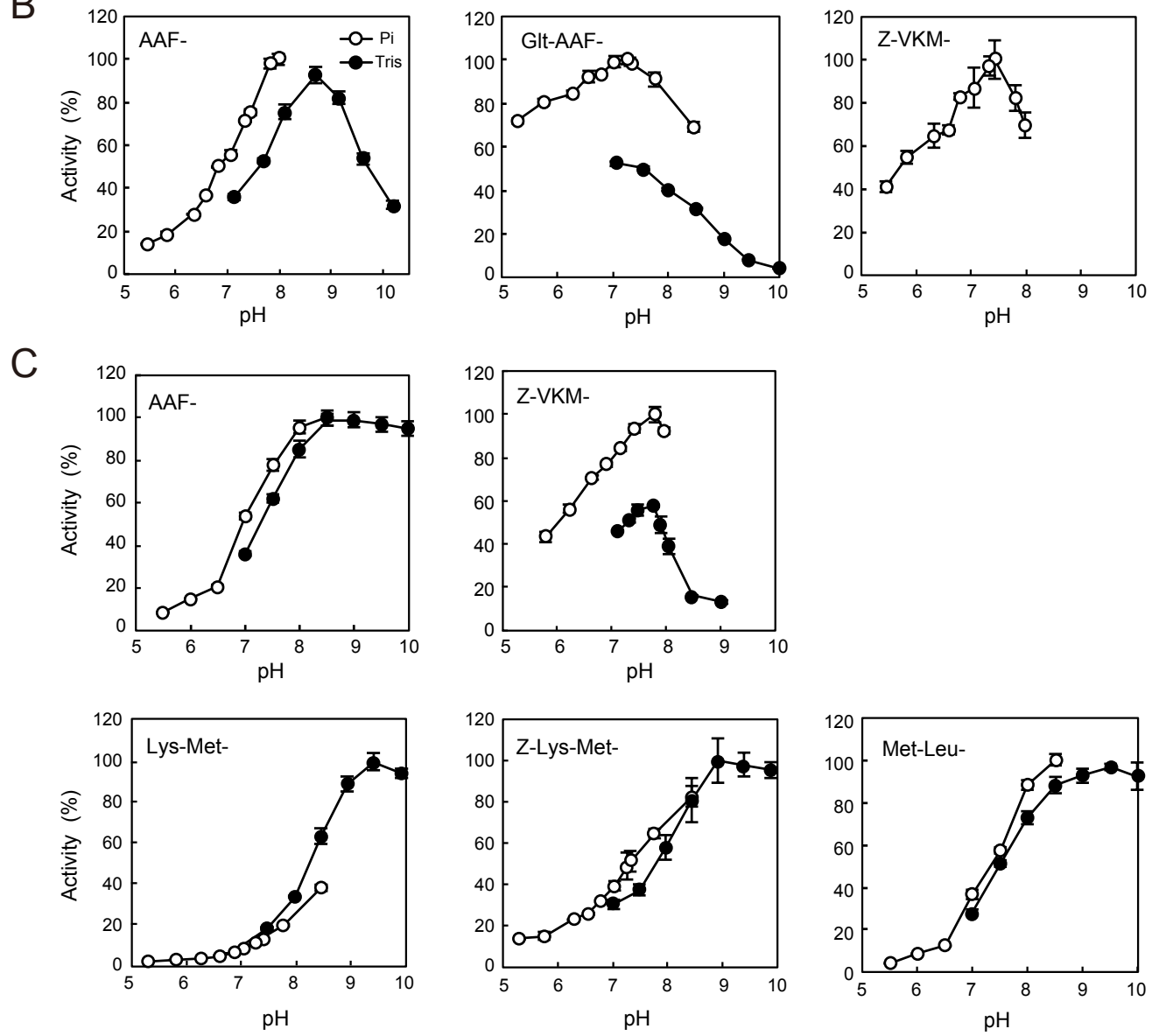

Supplemental Fig. S2. Expression and pH profiles of recombinant AOPs.

(A) Aliquots $(0.4 \mu \mathrm{g})$ of recombinant PgAOP (lane 1), LsAOP (lane 2), and BdAOP (lane 3) were separated on an SDS-PAGE gel. Lane M, low molecular-weight marker. Activities of (B) BdAOP and (C) LsAOP were determined with peptidyl MCA in $50 \mathrm{mM}$ sodium phosphate (open circle) or $50 \mathrm{mM}$ Tris-HCl (closed circle). The highest level of hydrolysis in each substrate was determined to be $100 \%$. Values are shown as the mean \pm S.D. $(n=3)$. 
A

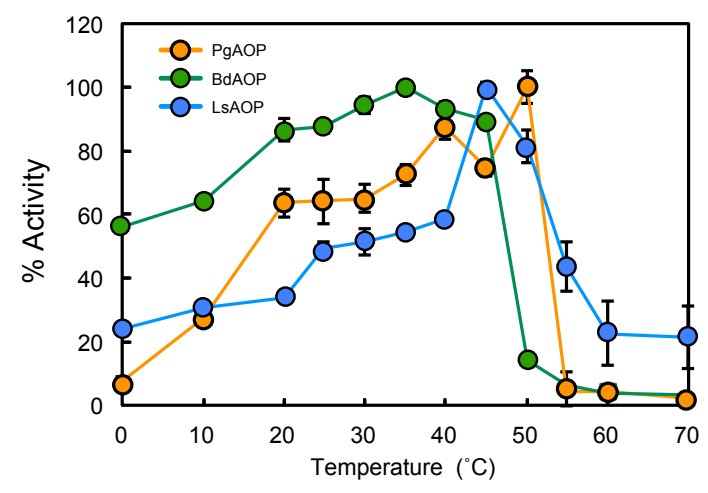

$\mathrm{B}$

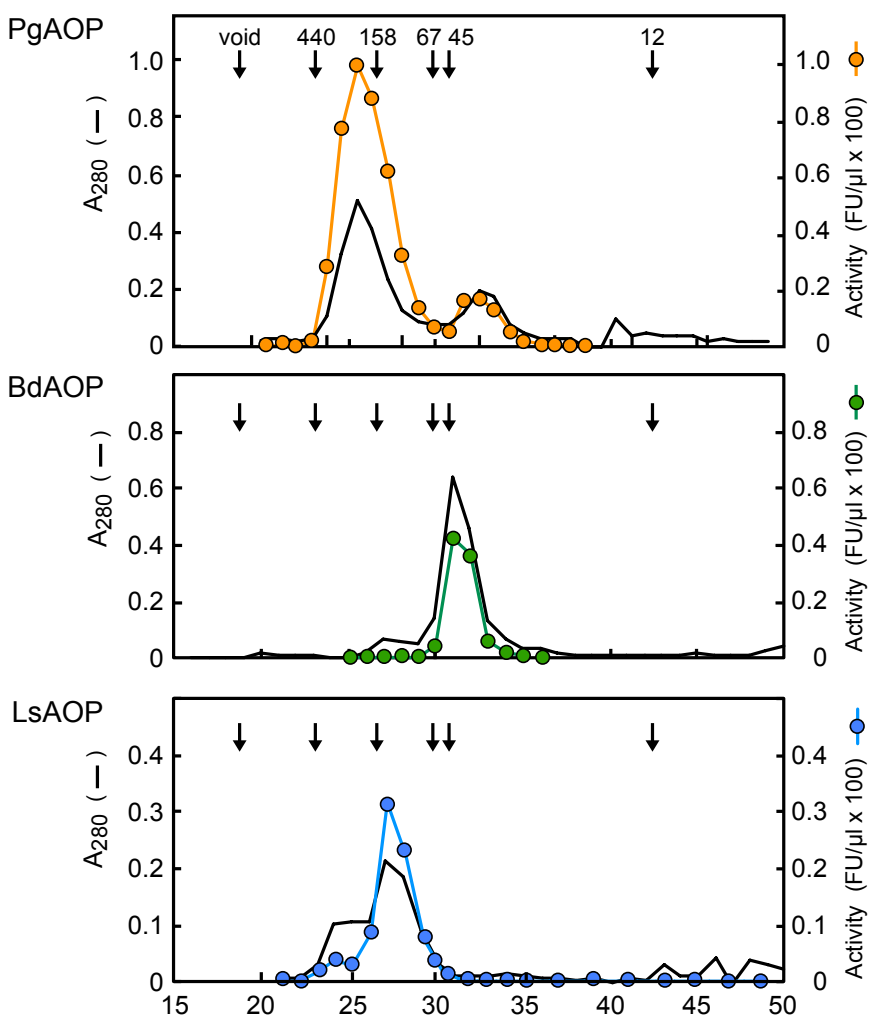

Supplemental Fig. S3. Heat dependence and molecular states of AOP.

(A) Recombinant AOP $(0.2 \mu \mathrm{g})$ was incubated with $20 \mu \mathrm{M}$-Lys-Met-MCA at $0-70{ }^{\circ} \mathrm{C}$ and the activity was determined. The activities of AOPs are presented to $100 \%$ at the maximal positions. (B) PgAOP, BdAOP, and LsAOP $(1.2 \mathrm{mg} / \mathrm{ml}, 0.3 \mathrm{ml})$ were subjected to size-exclusion HPLC with a Superdex200 10/300 column as described

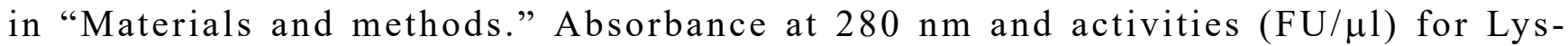
Met-MCA were determined. Molecular markers were run in parallel. 